\title{
Long-term outcomes after cosmetic customized prostheses and dermis fat graft in congenital anophthalmia: a retrospective multicentre study
}

\author{
Alessandra Claudia Modugno ${ }^{1} \cdot$ Antonio Giordano Resti $^{2} \cdot$ Giacomilde Mazzone $^{1} \cdot$ Caterina Moretti $^{1}$.

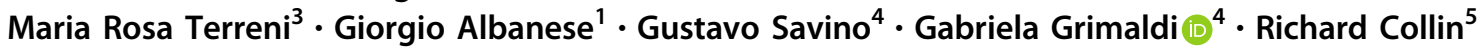

Received: 14 April 2018 / Accepted: 4 June 2018 / Published online: 24 July 2018

(c) The Royal College of Ophthalmologists 2018

\begin{abstract}
Purpose To evaluate long-term outcomes of progressively enlarging cosmetic customized prostheses (CCP) early after birth followed by dermis fat graft (DFG), as a strategy of socket rehabilitation in children with clinical congenital anophthalmia (CCA).

Methods Twenty patients with unilateral and two patients with bilateral CCA were enrolled. All patients were treated by inserting a CCP at the time of their first assessment which was then enlarged. Subsequently they underwent DFG. Differences in vertical palpebral aperture (VPA) and horizontal palpebral length (HPL), between affected and unaffected sides, were recorded at the first CCP fitting as well as before and after DFG. Satisfaction with cosmetic results, prosthetic retention, and complications rate were assessed. Magnetic resonance imaging of the orbit was performed in all patients before and after surgery.

Results A significant decrease in the difference between the normal and the anophthalmic side of both PA and HPL was found over follow-up. Both VPA and HPL differences decreased by $47.6 \%$ (10.5 mm, range 1-28 mm) and by $7.1 \%(5.8$ $\mathrm{mm}$, range $0-18 \mathrm{~mm}$ ), respectively. Satisfaction in terms of cosmetic outcomes proved to be very positive, being "very satisfied" for families and "satisfied" for physicians. Excellent retention of prostheses was observed in all cases.

Conclusions A rehabilitating strategy combining early CCP and further DFG proved to be a valuable approach in children with CCA, offering significant benefits in terms of socket expansion, prosthetic retention, psychological impact, and cosmetic outcomes.
\end{abstract}

\section{Introduction}

Eye development is a complex process that occurs early in embryogenesis and is regulated by a highly organized sequence of genetic events. Disruption of any of these events at whatever stage may result in a wide range of

$\triangle$ Alessandra Claudia Modugno

modugno@ocularistica.it

1 Ocularistica Italiana, Rome, Italy

2 Ophthalmology Unit, San Raffaele Scientific Institute, Milan, Italy

3 Pathology Unit, San Raffaele Scientific Institute, Milan, Italy

4 Institute of Ophthalmology, Università Cattolica del Sacro Cuore, Rome, Italy

5 Moorfields Eye Hospital, London, UK ocular congenital malformations, including anophthalmia, microphthalmia, and colobomas. Congenital anophthalmia is a rare malformation, with an estimated incidence of $0.18-0.4$ cases per 10,000 births [1-3].

The term congenital anophthalmia is often used incorrectly to define cases of absence of a clinically detectable eye. However, it is not unusual for magnetic resonance imaging (MRI) to show eye remnants within the orbit, outlining a condition more properly defined as clinical congenital anophthalmia (CCA). Anophthalmia can occur by itself or be associated with other malformations or with specific syndromes of various etiologies [4-10].

A full medical examination is mandatory to detect the presence of any related defect, such as ear, palate, heart, kidney, and brain malformations $[11,12]$. A comprehensive medical history, physical examination, family history, karyotyping, and genetic counseling are essential to determine the correct diagnosis and management [13, 14]. The 
diagnosis is usually based on clinical signs and MRI findings [15-17]. The main concern with partial or total absence of a normal-size eye within the orbit is the reduced degree of developmental stimulus for the bony orbit, eyelids, and socket soft tissues. As a result, a wide variability of potentially disfiguring orbital and eyelid malformations may develop and blindness may remarkably contribute to severe psychological and social impairment, especially in bilateral cases. To date, different approaches have been proposed to expand the socket soft tissues and bony orbit in order to achieve good prosthetic retention and maximize facial symmetry. Nevertheless, procedures that merely aim to enlarge the bony orbit, such as the early three-dimensional (3D) orbital osteotomy [18, 19], lead to an expansion of orbital bones and improvement of orbital symmetry, with no significant influence on the development of the conjunctival sac and eyelids [20]. Several specific devices have been reportedly used to produce socket volume expansion with subsequent stimulation of orbital growth, including solid shapes, hydrophilic expanders, and inflatable expanders [21, 22].

Indeed, management of anophthalmia requires a coordinated approach, involving both oculoplastic surgeons and ocularists, to fit prostheses shortly after birth. Expansion of congenital anophthalmic sockets has prompted different approaches over the past decades. Despite such a multidisciplinary strategy, CCA remains a very challenging issue and a wide consensus on its more effective treatment has not been achieved yet.

Hard implants are available but require multiple surgical procedures under general anesthesia to modify the implant size according to the socket volume difference [21, 22]. On the other hand, self-expanding socket hydrogel tissue expanders feature early expansion in the socket over the first $48 \mathrm{~h}$, occurring along the postero-anterior direction, with no influence on fornices depth [23-28].

Inflatable soft tissue expanders represent a further alternative for CCA although the direction of expansion has been found to be difficult to control and displacement of the conformer may occur early after inflation [21, 29, 30]. Furthermore, leakage and infection of the expanders have been described [29].

Tse et al. developed a particular orbital tissue expander in 2011, with promising results in terms of bone growth [31]. Nonetheless, the treatment does not include any measure to stimulate conjunctival socket expansion [31]. Standard conformers by themselves as prosthetic approach or followed by dermis fat graft (DFG) have turned out to be a useful option employed over the years [23]. In particular, cosmetic customized prostheses (CCP) are all the more indicated for CCA due to the advantage of fitting the patient's socket with customized prostheses.
The aim of our study was to evaluate the long-term developmental and cosmetic results of CCPs and DFG in children with CCA.

\section{Materials and methods}

This was a retrospective consecutive multicentre study (Ocularistica Italiana, Rome; Institute of Ophthalmology, Università Cattolica del Sacro Cuore, Rome; Ophthalmology Unit, San Raffaele Scientific Institute, Milan. Italy) of patients who were diagnosed with CCA between 2009 and 2014. Each patient underwent CCP placing at the first clinical assessment. Progressively enlarging CCPs were then changed fortnightly for the first 2 months and then 3weekly for the following 2 months. Afterwards, patients were followed up according to individual clinical findings every 2-6 months until DFG surgery.

Replacement of enlarging CCP was performed by an experienced ophthalmologist without anesthesia in an outpatient setting, employing a purpose-designed holder. Neither suture nor cyanoacrylate glue tarsorrhaphy were needed. Patients with CCA associated with large eyelid colobomas or orbital cysts were excluded from the study.

\section{DFG surgical technique}

The DFG was harvested from the upper outer quadrant of the gluteal region. The graft size was determined according to the volume and shape of the prosthesis. After the donor site was marked, an intradermal injection of saline solution was administered to facilitate the de-epithelialization of the skin from the graft. The DFG was then harvested. The deep layers of the donor site were closed with interrupted 4.0 vicryl sutures placed through the fatty and subcutaneous tissues. The skin was then closed with 5.0 vicryl sutures.

A conjunctival incision was made horizontally from the caruncle through the center to the lateral canthus. Blunt scissors were used to separate the endo-orbital tissues, bundles, and septa to allow the insertion of the graft. Four 6.0 absorbable sutures, one for each quadrant, were passed through the conjunctiva and Tenon's capsule before placing the implant. The DFG was then placed centrally in the socket. The conjunctival edge was sutured overlaying the dermis by $\sim 1 \mathrm{~mm}$. A custom-made clear conformer was inserted into the socket right after the surgery (Fig. 1) and a pressure dressing was applied for 2 days.

Postoperatively, a combination of steroid (betamethasone) and antibiotic (chloramphenicol) gel was given four times a day for a month. After one month, the conformer was replaced by a cosmetic CCP. MRI was performed preoperatively to evaluate the socket shape and the position 

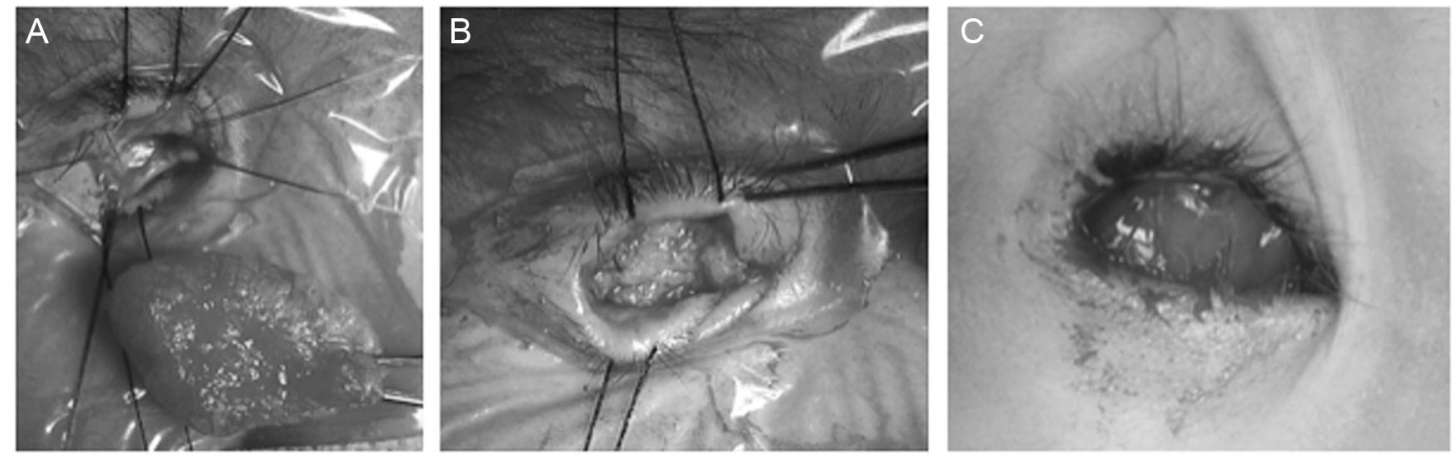

Fig. 1 DFG: surgical technique a, b, c. a The dermis fat graft is harvested from the upper outer quadrant of the gluteal region. $\mathbf{b}$ The

graft is positioned into the socket. c A clear customized conformer fitted after surgery
Fig. 2 Congenital bilateral malformation (right anophthalmia and left microphthalmia) a, b, c, d. a Esthetic result after CMC and prosthetic treatment on the right side. In the left side, no prosthesis was present at the time. b MRI shows the eye remnant and the tilted position of the prosthesis before DFG. c After combined treatment (CMC-DFG). The DFG was positioned below the eye remnant. A shell prosthesis was placed on the microphthalmic fellow eye. $\mathbf{d}$ MRI after DFG the prosthesis is properly positioned in the socket and remodeling of the fornixes
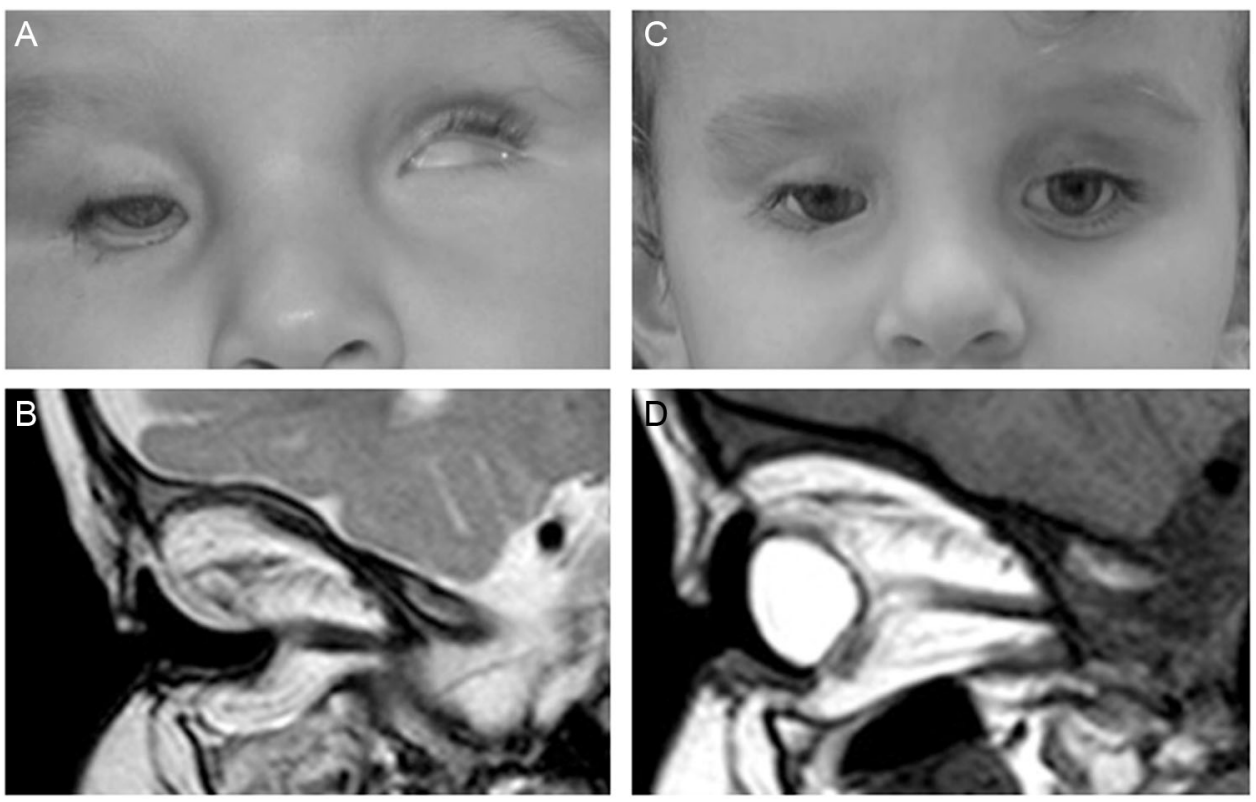

of the CCP in the socket as well as to detect the presence of any eye remnant within the orbit.

When MRI ruled out the presence of eye remnants within the orbit, a DFG was placed centrally in the socket. In five children, MRI detected the presence of an eye remnant located in the supero-temporal orbit. In two patients, the eye remnant was removed and replaced by the DFG. In the other three cases, the DFG was placed below the eye remnant (Fig. 2). Where present, hematoxylin and eosin staining of the biopsied remnant tissue was performed. Postoperative MRI was performed in all children starting 6 months after surgery to evaluate graft and prosthesis position.

Patients were followed up at 1 day, 1 week, 1 month, and 3 months postoperatively until the end of the first year.

Vertical palpebral aperture (VPA) and horizontal palpebral length (HPL) were measured in millimeters $(\mathrm{mm})$ in all patients. The difference between affected and unaffected sides was assessed only in unilateral CCA and was expressed in percentage (\%). Measurements were obtained by the same physician at three significant timepoints: at first $\mathrm{CCP}$ fitting (T0), preoperatively before DFG (T1) and at the last available follow-up (T2), respectively. Prostheses stability and position into the socket were carefully assessed at each follow-up. The integration of DFG, fornices depth, the presence of eyelid malpositions, and complications were also assessed at each examination.

Satisfaction of both families and physicians with the cosmetic outcome was appraised using a 0-10 scale questionnaire, with $0-4$ being classified as "dissatisfied", 5-7 as "satisfied", and 8-10 as "very satisfied".

The Institutional Review Board of the Università Cattolica del Sacro Cuore of Rome approved the study, which was carried out according to the tenets of the Declaration of Helsinki. The institutional review board reviewed and approved data management. Informed consent in writing, including an explanation of the clinical procedures and 
Table 1 Gender, age of first cosmetic customized prostheses, dermis fat graft and last postoperative follow-up

\begin{tabular}{|c|c|c|c|c|}
\hline Patients & Gender & $\begin{array}{l}\text { 1st CCP } \\
\text { months }\end{array}$ & $\begin{array}{l}\text { DFG } \\
\text { months }\end{array}$ & $\begin{array}{l}\text { Last follow-up } \\
\text { months }\end{array}$ \\
\hline 1 & M & 4 & 13 & 63 \\
\hline 2 & M & 2 & 16 & 53 \\
\hline 3 & $\mathrm{~F}$ & 1 & 28 & 90 \\
\hline 4 & $\mathrm{~F}$ & 3 & 17 & 62 \\
\hline 5 & M & 2 & 30 & 59 \\
\hline 6 & M & 1 & 20 & 17 \\
\hline 7 & M & 2 & 21 & 66 \\
\hline 8 & $\mathrm{~F}$ & 2 & 20 & 74 \\
\hline 9 & $\mathrm{M}$ & 1 & 36 & 39 \\
\hline 10 & M & 5 & 20 & 58 \\
\hline 11 & M & 3 & 19 & 74 \\
\hline 12 & $\mathrm{~F}$ & 1 & 22 & 92 \\
\hline 13 & $\mathrm{~F}$ & 4 & 14 & 67 \\
\hline 14 & M & 1 & 14 & 25 \\
\hline 15 & M & 1 & 9 & 26 \\
\hline 16 & M & 3 & 60 & 36 \\
\hline 17 & $\mathrm{~F}$ & 28 & 96 & 56 \\
\hline 18 & M & 5 & 84 & 62 \\
\hline 19 & $\mathrm{~F}$ & 6 & 48 & 16 \\
\hline 20 & M & 1 & 30 & 29 \\
\hline 21 & M & 1 & 18 & 37 \\
\hline 21 & $\mathrm{M}$ & 1 & 27 & 28 \\
\hline 22 & $\mathrm{M}$ & 1 & 72 & 27 \\
\hline 22 & M & 1 & 84 & 15 \\
\hline
\end{tabular}

$C C P$ cosmetic customized prostheses, $D F G$ dermis fat graft

potential complications of surgery, was obtained from the enrolled patients' parents. Photographs were taken in selected cases with parents' permission.

\section{Results}

Twenty patients presented with unilateral CCA, whereas CCA was bilateral in two cases. Patient demographics, timing of CCP and DFG, and total follow-up time are outlined in Table 1.

At the time of the first cosmetic CCP fitting, children's mean age was 3.3 months (range 1-6 months), with the exception of one patient who was examined for the first time at 28 months of age.

Mean age at the time of DFG was $34.1 \pm 25.1$ months, with the youngest case undergoing surgery 9 months after birth and the oldest being 8-year-old.

DFG was performed at an average age of 20.8 months (range 9-48 months) in 18 orbits. Five children (six orbits) underwent DFG at an older age, ranging from 4 to 8 years, owing to unrelated family and health circumstances. After the DFG procedure, an adequate filling of the socket was achieved in all children, with good prosthetic retention, deepening of the fornixes and eyelid expansion. The mean follow-up was $48.8 \pm 22.6$ months (range 15-92 months).

On preoperative MRI, the presence of a non-cystic lump located in the supero-temporal orbit was reported in five patients (Fig. 3a). In two cases, the remnant was removed and replaced by the DFG and histological examination revealed the presence of a rudimentary eye (Fig. 3b). In the other three cases, DFG was placed below the eye remnant.

One patient underwent surgical removal of the eye remnant and simultaneous replacement with the DFG. After 6 months, the child developed a lower lid entropion. MRI detected the DFG in the same place as the removed eye remnant in the superior orbit, along with an excess of fat growth. As a consequence, the prosthesis was pushed onwards and downwards, resulting in prosthesis tilting causing traction on the posterior lamella and consequent entropion. Therefore, a debulking of the fat excess was performed, allowing good prosthetic retention. To avoid this complication, the DFG was placed below the eye remnant in the remaining four cases, filling homogeneously the socket according to the MRI findings.

A significant decrease in the PA T0-T2 difference was observed in 19 patients with unilateral CCA (95\%). The average VPA difference dropped by $47.6 \%(10.5 \mathrm{~mm})$, ranging between 1 and $28 \mathrm{~mm}$, with the main difference reduction occurring before DFG (T0-T1).

The HPL T0-T2 difference decreased slightly over follow-up. A mean decrease of the HPL difference by $7.1 \%$ $(5.8 \mathrm{~mm})$ was observed, ranging between 0 and $18 \mathrm{~mm}$. The difference between T0-T1 was found to be higher than T1-T2, but still less remarkable compared with the VPA difference. In patients with bilateral CCA, an improvement of both VPA and HPL was detected over follow-up.

When assessing satisfaction with cosmetic outcomes, parents' mean score was 8.7 (very satisfied), whereas physicians' mean score was 7 (satisfied).

Neither socket nor fornix contraction were observed during follow-up, and no fat resorption occurred after DFG. A keloid on the gluteal scar was noted after 6 months in one child $(4.2 \%)$.

The esthetic appearance of all children improved after treatment. The best results in terms of prosthetic retention and cosmesis were achieved in those patients who underwent surgery between 12 and 24 months of age.

\section{Discussion}

The correct treatment of clinical congenital anophthalmia should aim to expand eyelids, fornices, socket soft tissues, 
Fig. 3 Eye remnant: radiological and histological findings a, b. a Magnetic resonance imaging revealing the eye remnant located supero-temporally in the orbit. a Histological specimen: rudimentary eye with thickened sclera (1), cyst filled with proliferated glial tissue (2), neural retinal (3), and choroidal detachments

(Haematoxylin-Eosin, 1×)
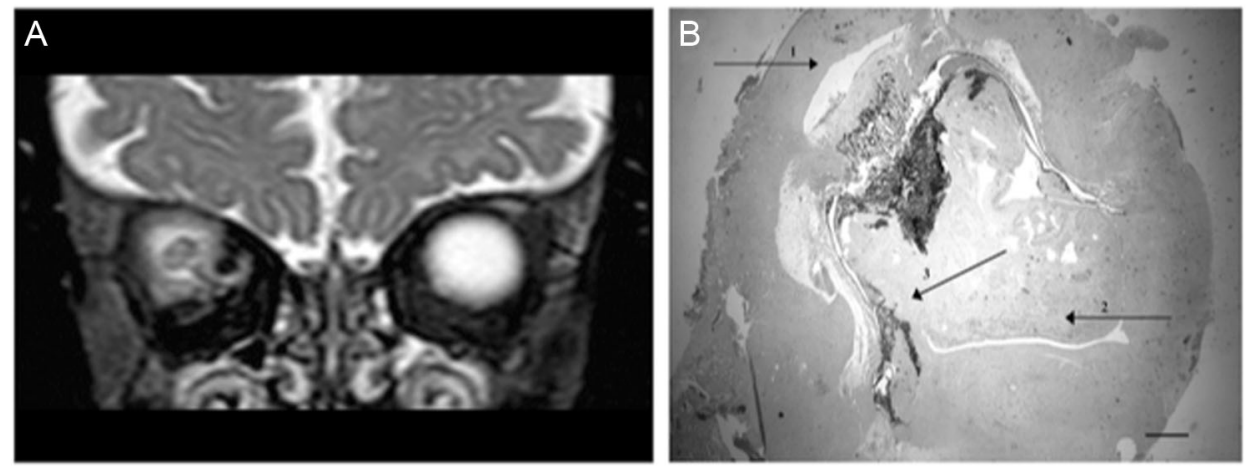

and orbital bones. This allows children to fit suitable prosthesis, minimizing facial asymmetry, and improving cosmetic appearance. Success or failure of this treatment will affect not only the child's appearance, but also his psychological and social development [31, 32].

Nevertheless, to date a widely recognized consensus on the most appropriate management of this condition is still lacking. Several strategies have been proposed but none of them succeeded in addressing all aspects of this complex malformation [21-31].

Hard silicon spheres require multiple general anesthetic and surgical procedures to change the size of the implants and do not yield a significant increase of socket lining. Selfexpanding hydrogel tissue expanders have been revisited recently as an alternative treatment to enlarge the volume of socket and/or orbital bones in congenital anophthalmia [2328]. These implants may be either socket hemispherical or orbital spherical shaped. The former hydrogel implants are placed in the conjunctival sac and increase their volume along a postero-anterior vector very quickly once in place. They will increase the conjunctival lining but when they become unstable they have to be replaced with a hard shell conformer to maintain the fornices $[13,21]$. The orbital volume is then increased with a DFG. The main limits of orbital spherical shaped implants are migration and extrusion, conjunctival erosion, and orbital inflammatory reaction [26].

Inflatable orbital tissue expanders seem to provide better bony orbit expansion, but do not expand the fornices, making it difficult to retain enlarging prostheses.

Although a similar approach using expandable conformers to increase palpebral aperture and fornices depth was described by Lamb in 1970 [33], these techniques do not provide early cosmetic rehabilitation, and do not fill the volume gap nor deepen fornices.

According to our experience, standard conformers result in a tilted position and are not suitable to fit sockets whose shape presents with dramatically shallow fornices. Conversely, our non-invasive approach allows patients to fit a cosmetic CCP within the first month of life with no need of anaesthesia.
Using CCPs leads not only to immediate familiar satisfaction in terms of cosmetic outcome [34] but also provides patients and families with the opportunity to immediately begin the rehabilitating pathway. Indeed, fitting a CCP at an early stage allows children to be part of their own social environment, easing potential family emotional distress. Above all, this strategy permits the expansion of socket soft tissues in order to correctly retain the prosthesis and to gain symmetry over the unaffected side. However, this first step allows to achieve a satisfactory expansion of the socket in its posterior portion, whereas fornices remain shallow and PA is still unsatisfactory.

The DFG allows to fill the socket with the correct volume, to increase the lining and to reshape and deepen fornices. It is known, indeed, that the increase of orbital volume promotes bone expansion, whereas the same outcome cannot be obtained vice versa [29]. As a result, prostheses can be placed forward along the antero-posterior axis, gaining facial symmetry. After the DFG, patients can easily wear a thinner prosthesis, which can be gradually increased in size (Fig. 4).

In our series neither fat absorption nor rejection were found, DFG being an autologous graft. The graft remained healthy over the entire length of follow-up, with an increase of its fat share according to socket development [35]. Furthermore, conjunctiva migrates from the wound edges, overlaying the dermal side of the graft and increasing the original socket lining. A debulking procedure was needed only in one case $(4.5 \%)$ although it is likely that the earlier the implant is positioned the greater the likelihood for the graft to grow. In many patients DFG have grown at puberty, although when performed at an older age DFG tends to a certain degree of fat atrophy [36].

Nonetheless, if the final volume was underestimated, the procedure can be safely repeated.

MRI clearly demonstrates the treatment process. Preoperatively, it helps the surgeon to place the DFG in the correct position into the socket, illustrating whether eye remnants are present or not. Postoperatively, it allows to evaluate graft position and the presence of deep fornices. It 

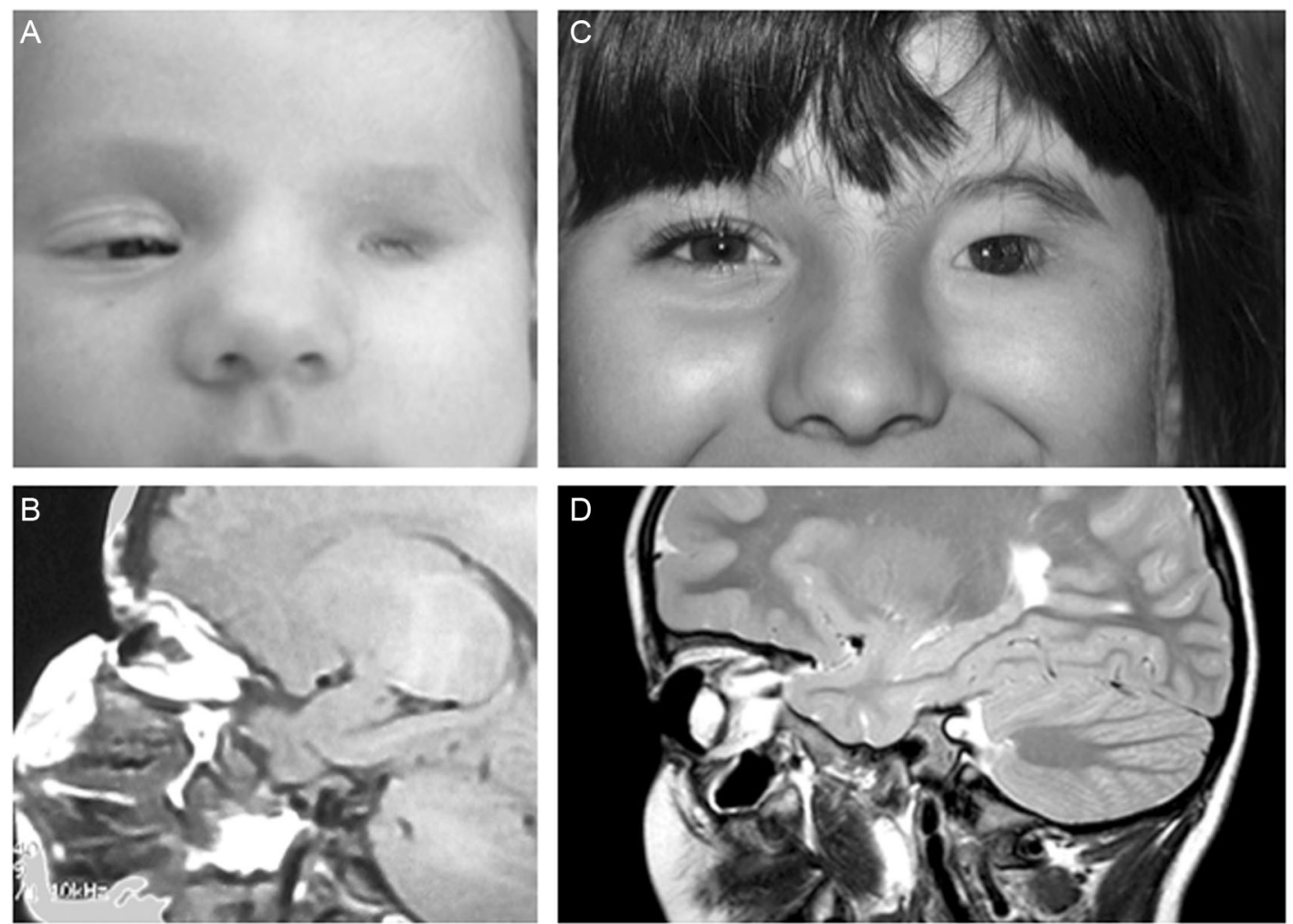

Fig. 4 Left congenital monolateral anophthalmia: cosmetic results $\mathbf{a}, \mathbf{b}$, c, d. a before treatment with CMC and customized prosthesis. b MRI before DFG shows a tilted prosthesis into the socket. $\mathbf{c}$ After CMC and

also shows how the prosthesis is thinner than before surgery and well placed within the socket. VPA and HPL were measured as indirect parameters of symmetry between the two sides. VPA difference between affected and unaffected sides experienced a significant decrease over the follow-up, leading to a relevant gain in facial symmetry. Treating children as soon as possible after birth, ideally within the first month of life, is mandatory to achieve such outcomes. Although a VPA increase might be argued to be mainly dependent on the size of the prostheses [21], deepening the fornices and providing the socket with a proper lining and volume are crucial steps in order to fit serially enlarging CCPs. Further expansion of the PA was then obtained through DFG. HPL difference featured a less-remarkable decrease, but proved still helpful to reach a higher degree of facial symmetry. The two patients with bilateral CCA were excluded from the difference assessment since it would have been unavoidably biased. However, a substantial increase of both VPA and HPL was observed in these cases, resulting in an acceptable degree of facial symmetry.

The initial severity of the malformation and children's age at the time of surgery deeply influences the final cosmetic and functional outcome. Despite the initial condition, children treated with DFG between 9 and 20 months of age achieved the best cosmesis, while those
DFG. d MRI after DFG shows the prosthesis properly positioned and the deepening of the fornixes

who underwent surgery at 4 years or older had poorer cosmetic results, even though parents still considered themselves very satisfied.

Volumetric rehabilitation of orbital bones and content by CCPs, DFG, and prosthetic treatment allows progressive molding and enlargement of the orbit during infancy, contributing to restoration of a certain degree of palpebral function. Nevertheless, the orbicularis muscle fibers are reduced in size and number, and tarsus appears very thin, often resulting in eyelid entropion. Further studies are needed to assess the most effective therapeutic strategy for the correction of entropion secondary to malformative congenital conditions, but the final outcome will be related to the degree of severity of the initial malformation.

Outcomes, sample size $(n=24)$ and long-term follow-up allow us to consider our method safe, effective, and repeatable.

\section{Conclusions}

This study confirms the importance of early use of cosmetic purpose-designed prostheses to improve psychological and social development of both children and their families as first step of the rehabilitating strategy. Expansion of the 
socket before any surgical treatment is essential. In our study, CCPs proved to work effectively and safely, allowing quick socket and fornices expansion with no need of anesthesia or tarsorrhaphy and with early cosmetic results. The use of hydrogel expanders remains a valid alternative for the first step of socket expansion. DFG is then key in order to expand socket lining and decrease volume deficit. As a result, patients achieve a remarkable degree of symmetry and satisfactory cosmesis.

\section{Summary}

\section{What was known before}

- Clinical congenital anophthalmia (CCA) is a rare malformative condition, characterized by partial or total absence of a normal-size eye within the orbit, causing developmental anomalies for the bony orbit, eyelids, and socket soft tissues.

- Management of CCA requires a coordinated approach, in order to expand the socket soft tissues and bony orbit to achieve good prosthetic retention and maximize facial symmetry.

- Hydrogel expanders are used effectively for the first step of socket expansion, followed by dermis fat graft (DFG).

\section{What this study adds}

- Progressively enlarging cosmetic customized prostheses early after birth followed by dermis fat graft represent an effective strategy of socket rehabilitation in children with CCA, allowing socket expansion and prosthetic retention, with satisfactory cosmetic outcomes and improvement of psychological and social development.

\section{Compliance with ethical standards}

Conflict of interest The authors declare that they have no conflict of interest.

\section{References}

1. Shaw GM, Carmichael SL, Yang W, Harris JA, Finnell RH, Lammer EJ. Epidemiologic characteristics of anophthalmia and bilateral microphthalmia among 2.5 million births in California, 1989-1997. Am J Med Genet A. 2005;13:36-40.

2. Kallen B, Tornqvist K. The epidemiology of anophthalmia and microphthalmia in Sweden. Eur J Epidemiol. 2005;20:345-50.

3. Morrison D, Fitzpatrick D, Hanson I, et al. National study of microphthalmia, anophthalmia and coloboma (MAC) in Scotland; Investigation of genetic aetiology. J Med Genet. 2002;39:16-22.
4. Fitzpatrick D, Van Heyningen V. Developmental eye disorder. Curr Opin Genet Dev. 2005;15:348-53.

5. Brunquell PJ, Papale JH, Horton JC, et al. Sex-linked hereditary bilateral anophthalmos. Pathologic and radiologic correlation. Arch Ophthalmol. 1984;102:108-13.

6. Marcus DM, Shore JW, Albert DM. Anophthalmia in the focal dermal hypoplasia syndrome. Arch Ophthalmol. 1990;108: 96-100.

7. Graw J. The genetic and molecular basis of congenital eye defects. Nat Rev Genet. 2003;4:876-88.

8. Mauri L, Franzoni A, Scarcello M, et al. SOX2, OTX2 and PAX6 analysis in subjects with anophthalmia and microphthalmia. Eur J Med Genet. 2015;58:66-70.

9. Fantes J, Ragge NK, Lynch SA, et al. Mutations in SOX 2 cause anophthalmia. Nat Genet. 2003;33:461-3.

10. Bardakjian T, Weiss A, Schneider A. Microphthalmia/Anophthalmia/ColobomaSpectrum. In: Adam MP, Ardinger HH, Pagon RA, et al., editors. GeneReviews ${ }^{\circledR}$ [Internet]. Seattle (WA): University of Washington, Seattle; 1993-2017.

11. Makhoul IR, Soudack M, Kochavi O, Guilburd JN, Maimon S, Gershoni-Baruch R. Anophthalmia-plus syndrome: a clinical report and review of the literature. Am J Med Genet A. 2007;143:64-8.

12. Abouzeid H, Boisset G, Favez T, et al. Mutations in the SPARCrelated modular calcium-binding protein 1 gene, SMOC1, cause waardenburg anophthalmia syndrome. Am J Hum Genet. 2011;88:92-8.

13. Ragge NK, Subak-Sharpe ID, Collin JR. A practical guide to the management of anophthalmia and microphthalmia. Eye (Lond). 2007;21:1290-300.

14. Schittkowski MP, Guthoff RF. Systemic and ophthalmological anomalies in congenital anophthalmic or microphthalmic patients. Br J Ophthalmol. 2010;94:487-93.

15. Al-Ghadyan AA, Kazi GQ, Cotlier E. Anophthalmos and first branchial arch defects. Ophthalmic Paediatr Genet. 1985;6: $169-78$.

16. Albernaz VS, Castillo M, Hudgins PA, Mukherji SK. Imaging findings in patients with clinical anophthalmos. AJNR Am J Neuroradiol. 1997; 18:555-61.

17. Phadke SR, Sharma AK, Agarwal SS. Anophthalmia with cleft palate and micrognathia: a new syndrome? J Med Genet. 1994;31:960-1.

18. Marchac D, Cophignon L, Achard E, Dufourmentel C. Orbital expansion for anophthalmia and micro-orbistism. Plast Reconstr Surg. 1977;59:486-91.

19. Tessier P, Rougier J, Hervouet F. Microphthalmias and congenital anophthalmias. In: Plastic surgery of the orbit and eyelids. Report of the French Society of Ophthalmology. New York: Masson Publishing, 1977. p. 184-90.

20. Eppley BL, Holley SH, Sadove AM, Experimental effects of intraorbital tissue expansion on orbitomaxillary growth in anophthalmos. Ann Plast Surg. 31:19-26.1993;

21. Tucker SM, Sapp N, Collin R. Orbital expansion of congenitally anophthalmic socket. Br J Ophthalmol. 1995;79:667-71.

22. Kennedy RE. The effect of early enucleation on the orbit in animals and humans. Trans Am Ophthalmol Soc. 1964;62:459-510.

23. Bernardino CR. Congenital anophthalmia: a review of dealing with volume. Middle East Afr J Ophthalmol. 2010;17:156-60.

24. Krastinova D, Kelly MB, Mihaylova M. Surgical management of the anophthalmic orbit, part 1: congenital. Plast Reconstr Surg. 2001;108:817-26.

25. Schittkowski MP, Guthoff RF. Injectable self inflating hydrogel pellet expanders for the treatment of orbital volume deficiency in congenital microphthalmos: preliminary results with a new therapeutic approach. Br J Ophthalmol. 2006;90:1173-7. 
26. Mazzoli RA, Raymond WR 4th, Ainbinder DJ, Hansen EA. Use of self-expanding, hydrophilic osmotic expanders (hydrogel) in the reconstruction of congenital clinical anophthalmos. Curr Opin Ophthalmol. 2004;15:426-31.

27. Hou Z, Yang Q, Chen T, Hao L, Li Y, Li D. The use of selfinflating hydrogel expanders in pediatric patients with congenital microphthalmia in China. JAAPOS. 2012;16:458-63.

28. Gundlach KK, Guthoff RF, Hingst VH, Schittkowski MP, Bier UC. Expansion of the socket and orbit for congenital clinical anophthalmia. Plast Reconstr Surg. 2005;116:1214-22.

29. Dunaway DJ, David DJ. Intraorbital tissue expansion in the management of congenital anophthalmos. $\mathrm{Br} \mathrm{J}$ Plast Surg. 1996;49:529-35.

30. Cepela MA, Nunery WR, Martin RT. Stimulation of orbital growth by the use of expandable implants in the anophthalmic cat orbit. Ophthalmic Plast Reconstr Surg. 1992;8:157-67.
31. Nalawade TM, Mallikarjuna RM, Anand BM, Shashibhusan MA, Reddy VS. Prosthetic rehabilitation of a pediatric patient with an ocular defect. Int J Clin Pediatr Dent. 2013;6:62-5.

32. Jurel SK, Talwar N, Chand P, Singh RD, Gupta DS. Customization of stock eye prosthesis for a pediatric patient by a simplified technique. Int J Clin Pediatr Dent. 2012;5:155-8.

33. Lamb VR. An expandable conformer. Int Ophthalmol Clin. 1970;10:903-6.

34. Avisar I, Norris JH, Quinn S, et al. Temporary cosmetic painted prostheses in anophthalmic surgery: an alternative to early postoperative clear conformers. Eye (Lond). 2011;25:1418-22.

35. Mitchell KT, Hollsten DA, White WL, O'Hara MA. The autogenous dermis-fat orbital implant in children. JAAPOS. 2001;5:367-9.

36. Heher KL, Katowitz JA, Low JE. Unilateral dermis-fat graft implantation in the pediatric orbit. Ophthal Plast Reconstr Surg. 1998;14:81-8. 